\title{
Human Papillomavirus Infection-Associated Adenoid Cystic Carcinoma of the Hard Palate
}

\author{
Arthur Minwoo Chung · Dong Il Sun ${ }^{1}$. Eun Sun Jung · Youn Soo Lee \\ Departments of Hospital Pathology and ${ }^{1}$ Otorhinolaryngology, College of Medicine, The Catholic University of Korea, Seoul, Korea
}

Adenoid cystic carcinoma (ACC) is a rare tumor of the head and neck region. It accounts for less than $1 \%$ of all head and neck malignancies and approximately $4 \%-10 \%$ of all salivary gland tumors. ${ }^{1}$ Unlike squamous cell carcinoma, p16 protein overexpression is not a common feature of ACC, especially with a co-existing human papillomavirus (HPV) infection. ${ }^{2}$ Here, we present a case of ACC of the hard palate associated with HPV infection.

\section{CASE REPORT}

A 55-year-old male patient presented with a 3.9-cm mass in the right hard palate, which appeared 3 months ago and had increased in size over time. The patient was otherwise healthy without a remarkable medical history. He was a nonsmoker and did not have a history of alcohol or other substance use disorders. Magnetic resonance imaging revealed a lobular contoured mass in the right hard palate, extending to the retromolar trigone and involving the pterygoid muscle and plates. The patient underwent a right inferior maxillectomy. The cut surface of the resected specimen showed a whitish gray and firm mass with irregular margins, measuring $3.9 \mathrm{~cm}$ in diameter (Fig. 1). The mass was involving the maxillary bone. Microscopically, the lesion showed poorly circumscribed infiltrates of tumor cells forming a cribriform pattern (Fig. 2A). There was no squamous dysplasia of the overlying surface epithelium. Small to medium bland cells with scant cytoplasm surrounded pseudocysts, and mitotic

Corresponding Author

Arthur Minwoo Chung, MD

Department of Hospital Pathology, College of Medicine, The Catholic University of Korea, 222 Banpo-daero, Seocho-gu, Seoul 06591, Korea

Tel: +82-2-2258-1613, Fax: +82-2-2258-1628, E-mail: minu0731@gmail.com

Received: April 5, 2016 Revised: June 10, 2016

Accepted: July 7, 2016 figures were rarely seen. Perineural invasion was commonly seen, as was venous invasion. Approximately $75 \%$ of tumor cells were positive for p16 (RTU, Roche, Branford, CT, USA) (Fig. 2B) on immunohistochemistry. The ductal cells were positive for CD117 (1:400, DAKO, Glostrup, Denmark) (Fig. 2C), whereas the myoepithelial cells were strongly positive for $\mathrm{p} 63$ (1:200, DAKO) (Fig. 2D). HPV detection and genotyping were performed using the BMT HPV 9G DNA kit provided by Biometrix Technology Inc. (Chuncheon, Korea). DNA was isolated from the tissue using the DNA extraction kit, and amplification was performed with a polymerase chain reaction mixture consisting of extracted target DNA, the primer set, and polymerase chain reaction premix. Hybridization was performed using an HPV DNA chip at room temperature for 30 minutes. HPV DNA hybridization was detected using the DNA Chip Scanner (ScanArray Gx, Perkin-Elmer Life and Analytical Science, Boston, MA, USA). In this case, the tumor tissue DNA was positive for HPV type 16. The resection margins were free of tumor. On the basis of these findings, the histopathological diagnosis was HPV-associated ACC. After discussion with the head and neck multidisciplinary team, the patient underwent postoperative radiation therapy. No recurrence was evident at 6-month follow-up.

\section{DISCUSSION}

HPV has been shown to be etiologically related to the development of 20\%-25\% of head and neck carcinomas. It has a strong predilection for the oropharynx, as up to $80 \%$ of nonkeratinizing oropharyngeal squamous cell carcinomas are related to HPV infection. ${ }^{3}$ The likelihood of detecting HPV outside of the oropharynx is very low, especially in the nasal cavity. ${ }^{4}$ Using 
an HPV DNA chip, we report the first case of ACC of the hard palate associated with HPV infection.

Although p16 protein overexpression can be detected in ACC of the head and neck, the staining pattern of p16 is mostly focal

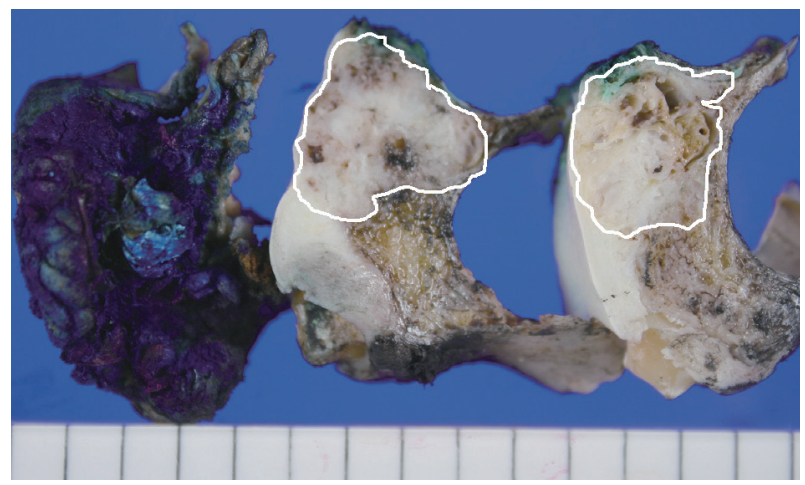

Fig. 1. On cut sections, an ill-defined, whitish gray mass (white circle), measuring $3.9 \mathrm{~cm}$ in diameter, involves the maxillary bone. (less than $75 \%$ of cells are stained), and the presence of co-existing HPV infection is rare. ${ }^{2,5}$ Even though p16 protein overexpression was observed in our case, we used an HPV DNA chip for confirmation.

The most important differential diagnosis to consider is HPVrelated carcinoma with adenoid cystic-like features due to its morphologic and immunohistochemical features. Its morphologic resemblance to ACC is based on architectural patterns, a dual population of cells, and immunohistochemical expression of myoepithelial markers. However, HPV-related carcinoma with adenoid cystic-like features has a predominantly solid growth pattern, and it is associated with squamous dysplasia of the overlying surface epithelium. ${ }^{1}$ Furthermore, HPV 33 is the most common high-risk HPV type in HPV-related carcinoma; however, HPV type 16 was detected in our case.

Because of their overlapping morphological features, basaloid squamous cell carcinoma must be distinguished from ACC. Un-
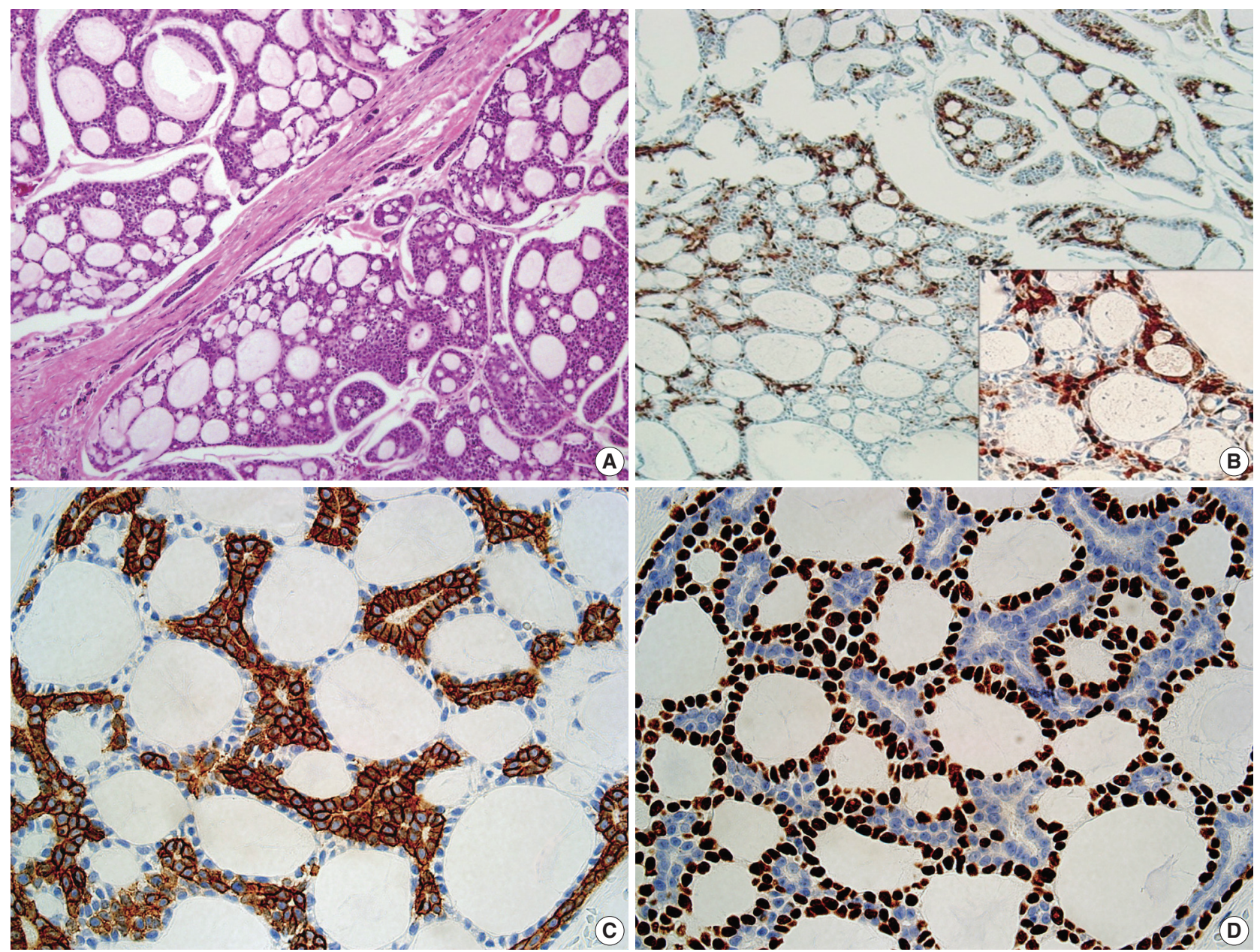

Fig. 2. Microscopic findings of the adenoid cystic carcinoma of the hard palate. (A) Adenoid cystic carcinoma with a cribriform pattern. (B) p16 protein expression is seen in 75\% of cells. The ductal cells are positive for CD117 (C), and the myoepithelial cells are positive for p63 (D). 
like basaloid squamous cell carcinoma, ACC usually lacks squamous differentiation. The most important differentiating characteristic of ACC is the presence of a myoepithelial cell component. In our case, the tumor has this dual population.

In summary, we present an HPV-associated ACC of the hard palate. HPV-associated ACC is extremely rare, and only two cases of HPV-related ACC arising in the nasal cavity have been reported. Here, we report the first documented case of ACC in the hard palate with the presence of $\mathrm{p} 16$ protein expression and co-existent HPV type 16 infection.

\section{Conflicts of Interest}

No potential conflict of interest relevant to this article was reported.

\section{REFERENCES}

1. Hwang SJ, Ok S, Lee HM, Lee E, Park IH. Human papillomavirus- related carcinoma with adenoid cystic-like features of the inferior turbinate: a case report. Auris Nasus Larynx 2015; 42: 53-5.

2. Boland JM, McPhail ED, Garcia JJ, Lewis JE, Schembri-Wismayer DJ. Detection of human papilloma virus and p16 expression in high-grade adenoid cystic carcinoma of the head and neck. Mod Pathol 2012; 25: 529-36.

3. Bishop JA, Guo TW, Smith DF, et al. Human papillomavirus-related carcinomas of the sinonasal tract. Am J Surg Pathol 2013; 37: 185-92.

4. Bishop JA, Ogawa T, Stelow EB, et al. Human papillomavirus-related carcinoma with adenoid cystic-like features: a peculiar variant of head and neck cancer restricted to the sinonasal tract. Am J Surg Pathol 2013; 37: 836-44.

5. Maruya S, Kurotaki H, Shimoyama N, Kaimori M, Shinkawa H, Yagihashi S. Expression of p16 protein and hypermethylation status of its promoter gene in adenoid cystic carcinoma of the head and neck. ORLJ Otorhinolaryngol Relat Spec 2003; 65: 26-32. 